\title{
Equilibrium properties of highly asymmetric star-polymer mixtures
}

\author{
Christian Mayer, Christos N. Likos, and Hartmut Löwen \\ Institut für Theoretische Physik II, \\ Heinrich-Heine-Universität Düsseldorf, \\ Universitätsstraße 1, D-40225 Düsseldorf, Germany
}

(Dated: June 16, 2018, submitted to Physical Review E)

\begin{abstract}
We employ effective interaction potentials to study the equilibrium structure and phase behavior of highly asymmetric mixtures of star polymers. We consider in particular the influence of the addition of a component with a small number of arms and a small size on a concentrated solution of large stars with a high functionality. By employing liquid integral equation theories we examine the evolution of the correlation functions of the big stars upon addition of the small ones, finding a loss of structure that can be attributed to a weakening of the repulsions between the large stars due to the presence of the small ones. We analyze this phenomenon be means of a generalized depletion mechanism which is supported by computer simulations. By applying thermodynamic perturbation theory we draw the phase diagram of the asymmetric mixture, finding that the addition of small stars melts the crystal formed by the big ones. A systematic comparison between the two- and effective one-component descriptions of the mixture that corroborates the reliability of the generalized depletion picture is also carried out.
\end{abstract}

PACS numbers: 61.20.-p, 61.20.Gy, 64.70.-p 


\section{INTRODUCTION}

Mixtures whose constituent particles show a high asymmetry in sizes are quite common in soft matter physics. As a matter of fact, all soft-matter systems are at least two-component mixtures, as they are typically suspensions or dispersions of mesoscopically-sized colloidal particles in a microscopic solvent. For many practical purposes, though, it suffices to model the solvent as a continuous medium and then an effective, one-component description of the suspended colloidal particles is sufficient. The phenomenology is much richer when more than one species of colloids is dispersed in the solvent and also there the asymmetry in the sizes of the two kinds of colloidal particles can be much higher than the one encountered in atomic or molecular fluids. In the recent past, a great deal of attention has been paid to the investigation of model colloid-polymer mixtures, in which the two species are hard colloidal spheres and soft, flexible polymer chains [1]. The bulk of the theoretical analysis of such systems is carried out within the framework of the Asakura-Oosawa (AO) model [2, 3, 4], in which the polymers are modeled as ideal, interpenetrating spheres that experience a hard repulsion towards the colloids. Another popular system that has attracted a lot of attention recently are binary hard-sphere mixtures of various size ratios [5, ㅁ, 7, 8, 9]. In both of those cases, attention is usually focussed on the influence of the smaller component on the structural and phase behavior of the larger one [10]. Demixing phase transitions and their competition to the crystallization transition of the large hard spheres have been an issue of intensive investigations in the past [11, 12, 13, 14, 15, 16, 17, 18] with current research steering in the direction of the study of interfacial and wetting properties of such mixtures 19, 20, 21, 22, 23, 24, 25, 26], as well as the influence of the additives on the vitrification transition of the hard spheres [27, 28, 29, 30, 31, 32, 33].

A convenient concept that has helped shed light into the phenomenology of such asymmetric mixtures is that of the effective, depletion interaction between the hard spheres, which is mediated by the smaller component [34, 35]. In the case of the AO-mixture, the depletion interaction is purely attractive and has the range of the size of the added polymer. For binary hard-sphere mixtures, the effective depletion potential displays oscillatory behavior due to correlation effects [36, 37]. Interpolating between the two extremes of ideal and hard additives are star polymers of varying functionality, whose depleting effects on hard spheres have been investigated both theoretically [38, 39] and experimentally [40]. 
The notion of depletion is almost exclusively invoked whenever the large particles are hard colloids. Nevertheless, it can be expanded in its interpretation to account for the modification of the properties of the large particles in the presence of smaller ones also for arbitrary kinds of interactions between the constituent particles. There is relatively little done in this direction, however, with the exception of the derivation of effective potentials in Yukawa mixtures [41] and in mixtures of star polymers and linear chains [42]. In the last case, it has been shown that the depletion mechanism of the chains on the stars can account for the experimentally observed melting of the star-polymer gel upon addition of linear polymer. In this paper, we turn our attention to two-component mixtures in which all particle species interact by means of soft potentials and, in particular, to mixtures of two kinds of star polymers: large ones with a high number of arms and small ones will a low arm number. All species interact via logarithmic-Yukawa pair potentials. We find that in this case the depletion mechanism of the small stars on the big ones has the effect of reducing the repulsive potential between the latter and thus it brings about a melting of the colloidal crystal formed by the large stars. Concomitant to this effect is a partial loss of correlations between the centers of the big stars, manifested in a drastic lowering of the peak height of their partial structure factor. Upon addition of a sufficiently large quantity of depleting agents, even an effective attraction between the large stars shows up, resulting in a demixing spinodal between the two species.

The rest of the paper is organized as follows: In Sec. II we present the pair potentials and the full, two-component description of the mixture, examining the effects of the depletants on the structural correlations of the big stars. In Sec. IIII, we formally trace out the small stars and examine the resulting effective, one-component interactions between the big ones. This effective potential is employed, in turn, in order to draw the phase diagram of the system in Sec. IV] where thermodynamic perturbation theory is used for the calculation of the Helmholtz free energies of the fluid and solid phases. In Sec. $\nabla$ we carry out a comparison between the one- and full two-component descriptions of the mixture and demonstrate the validity of the former, whereas in Sec. VT we summarize and draw our conclusions. 


\section{TWO-COMPONENT DESCRIPTION}

We consider binary mixtures of star-polymers which differ in terms of their sizes and arm numbers (functionalities). The system consists of $N_{1}$ stars of corona diameter $\sigma_{1}$ and functionality $f_{1}$ and $N_{2}$ stars, characterized by $\sigma_{2}$ and $f_{2}$, in a volume $V$. We first calculate the properties of the binary fluid. To obtain information about the pair correlations between the constituent particles, we describe the system using the full two-component picture for the mixture of the two different star-polymer species. The structural quantities we calculate are used as input for the mapping onto an effective one-component system in Sec. III We define the size ratio of the different species as $q=\sigma_{2} / \sigma_{1}<1$. Let $\rho_{i}=N_{i} / V(i=1,2)$ be the partial number densities of the two species.

We start from the effective pair potentials between the mesoscopic particles, having traced out the monomer and solvent degrees of freedom. The effective interaction between the starpolymers diverges logarithmically with the center-to-center distance $r$ as $r \rightarrow 0$, as derived by Witten and Pincus [43]. A full expression for identical star-polymers, which is valid for all star separations, has been derived and verified by neutron scattering and monomer resolved molecular simulation [44, 45]. The pair potential is given by an ultrasoft interaction which shows logarithmic behavior for small distances and an exponential Yukawa-type decay at large star-star separation [44, 46].

In the case of mixtures we need an expression for the effective interaction between star polymers in an athermal solvent that differ in their sizes $\sigma_{1}, \sigma_{2}$ [70] and functionalities $f_{1}$, $f_{2}$, as a function of their center-to-center separation $r$. In this work we use the effective pair potential which was put forward by means of field-theoretical arguments and confirmed by molecular dynamics computer simulations in Ref. [47], namely:

$$
\beta V_{i j}=\Theta_{i j} \begin{cases}-\ln \left(\frac{r}{\sigma_{i j}}\right)+\frac{1}{1+\sigma_{i j} \kappa_{i j}} & \text { for } r \leq \sigma_{i j} \\ \frac{1}{1+\sigma_{i j} \kappa_{i j}}\left(\frac{\sigma_{i j}}{r}\right) \exp \left(\sigma_{i j} \kappa_{i j}-r \kappa_{i j}\right) & \text { else }\end{cases}
$$

where $\sigma_{i j}=\left(\sigma_{i}+\sigma_{j}\right) / 2,1 / \kappa_{i j}=\sigma_{i} / \sqrt{f_{i}}+\sigma_{j} / \sqrt{f_{j}}$ and

$$
\Theta_{i j}=\frac{5}{36} \frac{1}{\sqrt{2}-1}\left[\left(f_{i}+f_{j}\right)^{3 / 2}-\left(f_{i}^{3 / 2}+f_{j}^{3 / 2}\right)\right] .
$$

Moreover, $\beta=\left(k_{\mathrm{B}} T\right)^{-1}$ is the inverse temperature, with $k_{\mathrm{B}}$ being Boltzmann's constant. Since all three interactions are purely entropic, the $\beta V_{i j}(r)$ are independent of the tempera- 
ture. For $i=j$ the potential reduces to the interaction of identical star-polymers which was introduced in [44]. In what follows, we fix the functionality of the large stars to $f_{1}=263$ in order to make contact with recently performed experiments [42] in which smaller polymeric entities were used as additives in gelated solutions of the large stars in order to examine their overall influence on the rheology of the mixture. This functionality is large enough for the star polymers to crystallize into a fcc-structure roughly at their overlap concentration [46]. For the small stars, we considered functionalities $f_{2}=16$ and 32 and size ratios $q$ in the range between 0.1 and 0.3 .

The pair structure of the mixture can now be calculated using the Ornstein-Zernike (OZ) equations for binary mixtures together with the two-component Rogers-Young (RY) closure. The pair correlations of the system are described by three independent total correlation functions $h_{i j}(r), i \leq j=1,2$, since the symmetry with respect to the exchange of indices dictates $h_{i j}(r)=h_{j i}(r)$ for $i \neq j$. In addition, we have the same number of direct correlation functions $c_{i j}(r)$. The Fourier transforms of these quantities are denoted by $\tilde{h}_{i j}(k)$ and $\tilde{c}_{i j}(k)$, respectively.

For multicomponent mixtures, the OZ relation takes the form [34, 48]

$$
\tilde{\mathbf{H}}(k)=\tilde{\mathbf{C}}(k)+\tilde{\mathbf{C}}(k) \cdot \mathbf{D} \cdot \tilde{\mathbf{H}}(k),
$$

where $\tilde{\mathbf{H}}(k)$ and $\tilde{\mathbf{C}}(k)$ are symmetric $\nu \times \nu$ matrices with

$$
[\tilde{\mathbf{H}}(k)]_{i j}=\tilde{h}_{i j}(k) \quad \text { and } \quad[\tilde{\mathbf{C}}(k)]_{i j}=\tilde{c}_{i j}(k) .
$$

$\mathrm{D}$ is a diagonal $\nu \times \nu$ matrix with

$$
[\mathbf{D}]_{i j}=\rho_{i} \delta_{i j}
$$

From Eq. (3) we obtain three independent equations for the six unknown functions $\tilde{h}_{i j}(k)$ and $\tilde{c}_{i j}(k), i, j=1,2$. In order to obtain a solvable system, we need three additional closure equations between these functions. The Rogers-Young closure for multicomponent mixtures reads as $[34]$

$$
g_{i j}(r)=\exp \left[-\beta V_{i j}(r)\right]\left[1+\frac{\exp \left[\gamma_{i j}(r) f_{i j}(r)\right]-1}{f_{i j}(r)}\right],
$$

where $g_{i j}(r)=h_{i j}(r)+1, \gamma_{i j}(r)=h_{i j}(r)-c_{i j}(r)$ and $V_{i j}(r)$ is the pair interaction between particles of species $i$ and $j$. The "mixing function" $f_{i j}(r)$ is defined as

$$
f_{i j}(r)=1-\exp \left(-\alpha_{i j} r\right)
$$



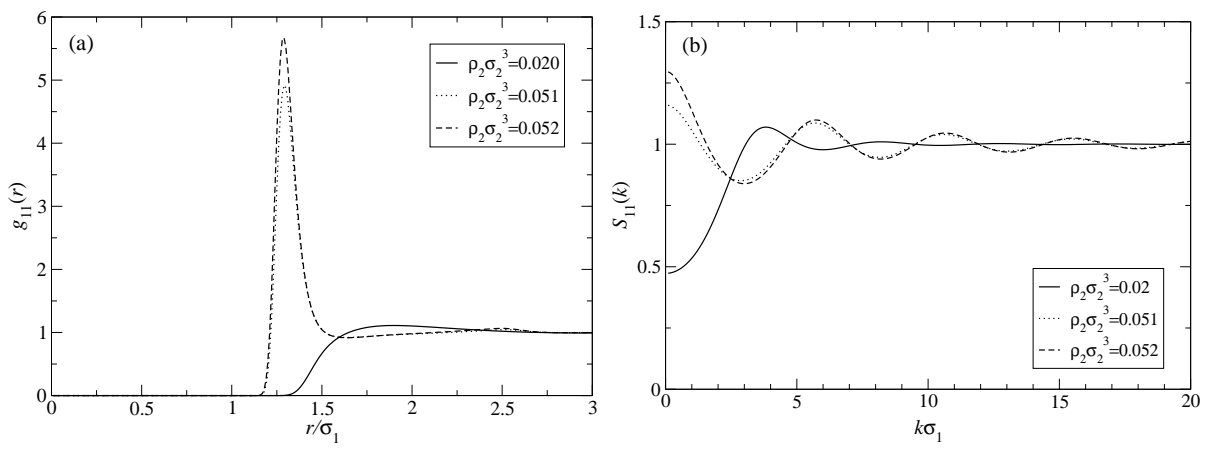

FIG. 1: The partial (a) radial distribution functions and (b) static structure factors for species 1 (big stars) in a mixture with small ones. The density of species 1 is $\rho_{1} \sigma_{1}^{3}=0.05$. The structure has been calculated using the two component OZ equations with the RY closure. The plotted lines are for different densities $\rho_{2}$, as indicated in the legend. In this case, $q=0.1$ and $f_{2}=32$. The partial structure factor grows for $k \rightarrow 0$ with increasing $\rho_{2}$, as we approach the demixing spinodal line of the system.

Usually, the same self-consistency parameter $\alpha=\alpha_{i j}$ is used for all components of the mixture. This allows to fulfill one thermodynamic consistency requirement, namely the equality between the "virial" and "fluctuation" total compressibilities of the mixture. Multiparameter versions have also been proposed [6], invoking thermodynamic consistency for the partial compressibilities of each species. For $\alpha \rightarrow 0$ Eq. (6) reduces to the Percus-Yevick (PY) and for $\alpha \rightarrow \infty$ to the hypernetted chain (HNC) multicomponent closures. When dealing with star-polymers, which feature a soft repulsion of relatively short range, neither the PY nor the HNC closure are adequate to capture the details of the correlation functions with high accuracy, therefore employing the full RY closure is essential [49].

In our work we solve the OZ equation with the RY-closure [Eqs. (3)-(17)] for the twocomponent mixture. The effective interactions between the star polymers are given by Eq. (11). The thermodynamic consistency of the RY closure was obtained by using a single parameter $\alpha$. The structure of the binary mixture can be described either by the partial radial distribution functions $g_{i j}(r)=h_{i j}(r)+1$ in real space or by the three partial static structure factors $S_{i j}(k)=\delta_{i j}+\sqrt{\rho_{i} \rho_{j}} \tilde{h}_{i j}(k)$ in wavenumber space. The structure factors are relevant in comparing with experiments, because they can be measured via scattering techniques.

In Fig. 1(a) we show results for the radial distribution function $g_{11}(r)$ between the large 

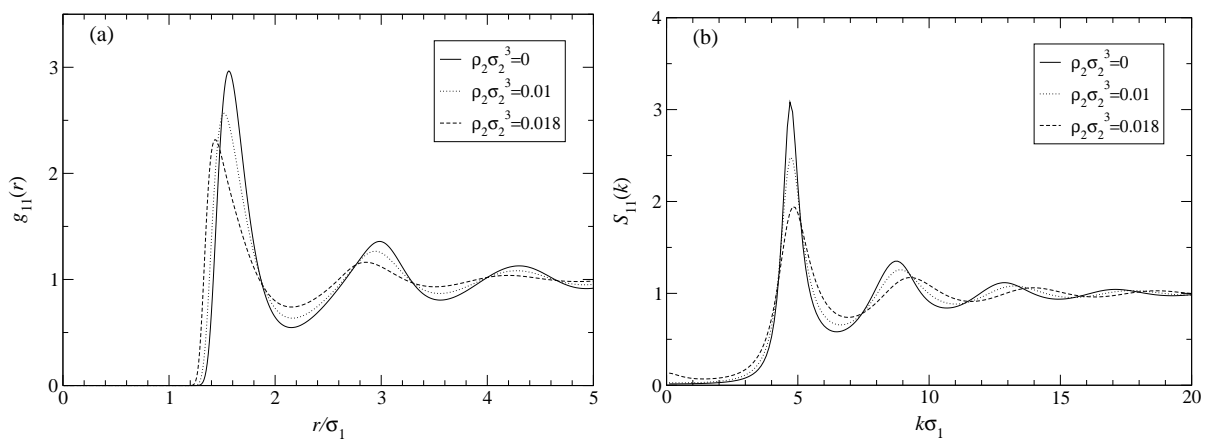

FIG. 2: Same as Fig. 1 but now for big star density $\rho_{1} \sigma_{1}^{3}=0.3$. By increasing the density of the smaller component, the structure of the fluid diminishes.

stars in a dilute solution, and its evolution upon increasing the concentration of small additives with $f_{2}=32$ and $q=0.1$. Although for very small concentration of smaller stars the function $g_{11}(r)$ has a relatively structureless shape, it rapidly develops a pronounced peak when $\rho_{2}$ is further increased. This is a first indication of clustering of big stars, which has its physical origin in some effective attraction induced by the small component. One physically expects that when this attraction becomes sufficiently strong, a demixing transition between the two species will take place. This hypothesis is corroborated by the evolution of the corresponding structure factor $S_{11}(k)$, shown in Fig. 1(b). A fluid-fluid demixing binodal is indicated by the divergence of all partial structure factors in the long-wavelength limit $k \rightarrow 0$. As can be seen in Fig. 1(b), a growth of the $k \rightarrow 0$-limit occurs upon increasing $\rho_{2}$. The existence of a demixing spinodal will be confirmed in Sec. IV where we draw the phase diagram of the mixture.

We now examine the effect of the additives at the complementary regime of high concentration of large stars, and in particular slightly above their overlap concentration, in which the latter are in a thermodynamically stable crystalline state [46] or in a dynamically arrested gel state [50]. We derive the partial structure factor $S_{11}(k)$ of the (metastable) fluid in the absence of small stars and monitor its evolution as $\rho_{2}$ is increased. Representative results are shown in Fig. 2. In Fig. 2(a), already a loss of correlations in real space can be discerned, as witnessed by the broadening and lowering of the coordination peaks in $g_{11}(r)$. Moreover, the large stars approach closer to each other upon an increase of $\rho_{2}$, an effect that can be interpreted as a weakening of the strength of their mutual repulsion. As can be seen in Fig. 2(b), the principle peak height of the structure factor of species 1 diminishes as 
the density of the smaller component is increased. The Hansen-Verlet criterion [51] states that a fluid solidifies when the maximum of the structure factor exceeds the threshold value $S_{\mathrm{th}}\left(k_{\max }\right)=2.85$. Therefore, the diminishing of structure in the system is a first indication for the melting of the crystal of big star-polymers by addition of the smaller species. In Fig. 2(b) it can be seen that the first peak of the structure factor is bigger than 3 for $\rho_{2} \sigma_{2}^{3}=0$. Already small densities of the smaller component lead to a drastic decline of the peak height, a finding that is in line with recent experimental and theoretical results on mixtures of star polymers with linear chains [42].

\section{EFFECTIVE ONE-COMPONENT DESCRIPTION}

We now wish to put the assumptions regarding the influence of the additives on the effective interaction of the big stars into a concrete test, by calculating an effective potential $V_{\text {eff }}(r)$ between the latter in the presence of the former. To this end, we carry out the mapping of the two-component system onto an effective one-component description, in which the degrees of freedom of the smaller star-polymers have been traced out. The interactions cause spatial correlations of the density of small stars in the vicinity of two big ones, influencing thereby the shape of the resulting generalized depletion interaction. There are different methods to obtain these effective interactions. All of them omit many-body forces and reduce the interaction to an additive pair potential, we will confirm however that manybody effects only play a minor role.

Instead of the so-called 'system representation', in which the two densities $\rho_{1}$ and $\rho_{2}$ in the mixture are given, we now switch into the more convenient 'reservoir representation' $\rho_{1}$ and $\rho_{2}^{\mathrm{r}}$. Since the effective interaction between the large stars depends rather on the

chemical potential $\mu_{2}$ of the small ones rather than on their density $\rho_{2}$, this description is more convenient [9]. The reservoir is a system consisting of pure small stars and their density there, $\rho_{2}^{\mathrm{r}}$, is determined by the requirement that the partial chemical potentials $\mu_{2}$ in the real system and $\mu_{2}^{\mathrm{r}}$ in the reservoir are equal. Clearly, due to the finite value of the density $\rho_{1}$ in the system, it must hold $\rho_{2} \neq \rho_{2}^{\mathrm{r}}$. The mapping between the two densities, depending parametrically on the big star density $\rho_{1}$, will be carried out in Sec. $\nabla$ 

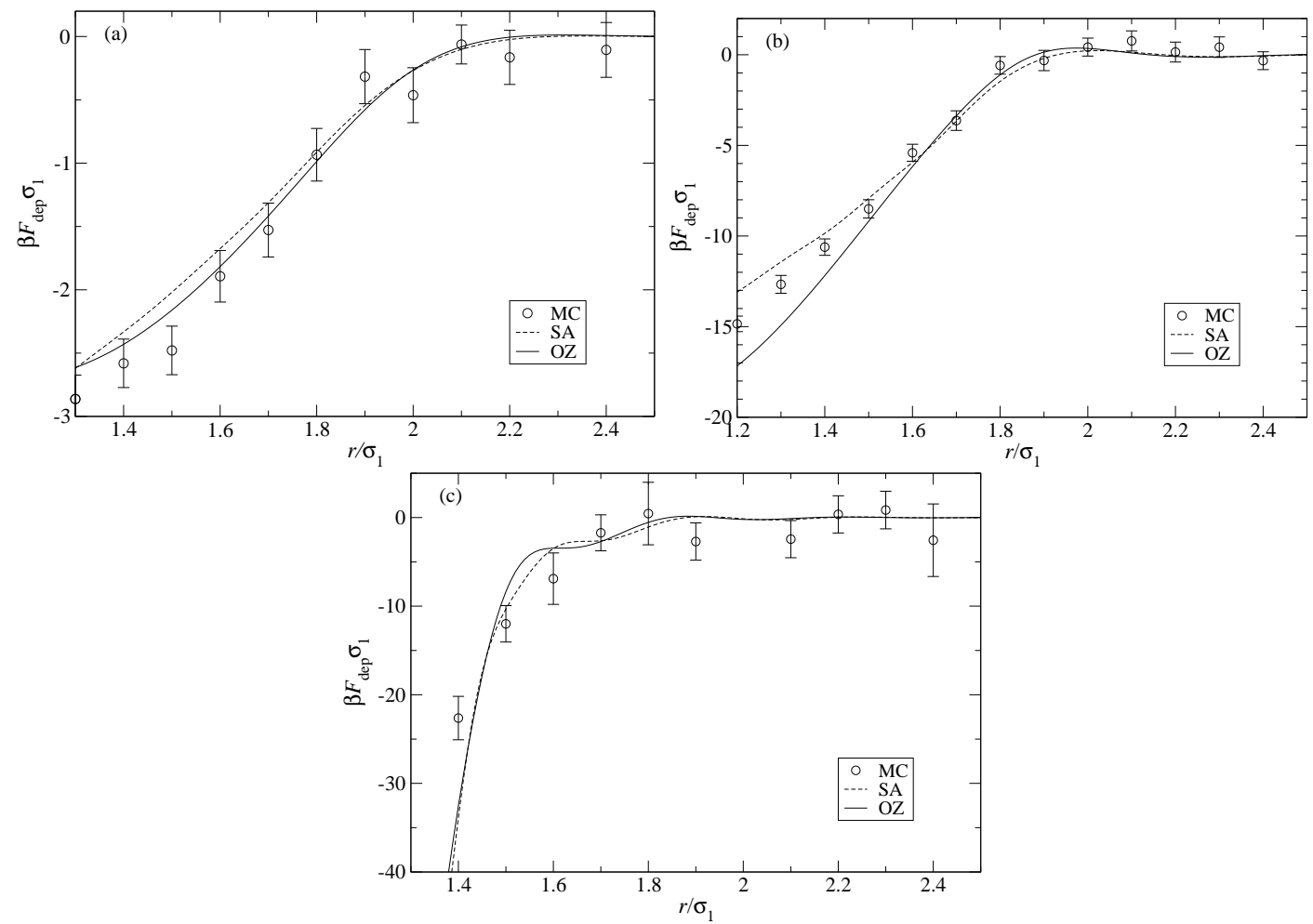

FIG. 3: Comparison of Monte Carlo simulation (MC), inversion of Ornstein-Zernike equation (OZ), and superposition approximation (SA) results for the depletion forces between the big stars. (a) Results for $q=0.3, f_{2}=32$ and $\rho_{2}^{\mathrm{r}} \sigma_{2}^{3}=0.027$; (b) $q$ and $f_{2}$ same as in (a) but $\rho_{2}^{\mathrm{r}} \sigma_{2}^{3}=0.081$; (c) for $q=0.2, f_{2}=32$ and $\rho_{2}^{\mathrm{r}} \sigma_{2}^{3}=0.08$.

\section{A. Simulations}

The most accurate way to obtain the effective interactions between the big star-polymers is a computer simulation of the mixture [54, 55, 56]. We place two big stars of species 1 at fixed positions $\mathbf{R}_{1}$ and $\mathbf{R}_{2}$ along the diagonal of the simulation cube, so that their common center coincides with the center of the cube. They are surrounded by the smaller species that move according to the forces dictated by the effective interactions of Eq. (11). Since we have only two big star-polymers in our simulation box, the density is $\rho_{1} \rightarrow 0$. Therefore the simulation provides directly the sought-for effective force as a function of the reservoir density $\rho_{2}^{\mathrm{r}}$.

We use standard NVT Monte Carlo simulation with periodic boundary conditions and minimum image convention. The length of the cubic simulation box is $L=5 \sigma_{1}$, so that the number of small stars in the simulation results which are shown in Fig. 3 is between 125 
and 1250. For each particle up to 5 million Monte Carlo steps are calculated, where the maximum displacement of the particles is chosen in such a way that half the steps will be accept. The force is then measured after every 1000 simulation steps. Due to the presence of the second big star, the density distribution around each star is not spherically symmetric. This leads to an average nonvanishing force between them, which is mediated by the small star-polymers and parallel to the vector $\mathbf{R}_{12}=\mathbf{R}_{2}-\mathbf{R}_{1}$. Due to the symmetry of the system, the components perpendicular to $\mathbf{R}_{12}$ have to vanish. The force $\mathbf{F}_{1}$ acting on the particle at $\mathbf{R}_{1}$ can then be calculated by averaging over the simulation results, namely:

$$
\mathbf{F}_{1}\left(R_{12}\right)=\left\langle-\sum_{j=1}^{N_{2}} \nabla_{\mathbf{R}_{1}} V_{12}\left(\left|\mathbf{R}_{1}-\mathbf{r}_{j}\right|\right)\right\rangle_{\mathbf{R}_{12}}
$$

where $\mathbf{r}_{j}$ are the star-polymer positions of species 2 and $\langle\cdots\rangle_{\mathbf{R}_{12}}$ denotes the statistical average, taken under the constraint of constant $\mathbf{R}_{12}$. Clearly, the effective force satisfies the relation $\mathbf{F}_{2}\left(R_{12}\right)=-\mathbf{F}_{1}\left(R_{12}\right)$. We further define the depletion force $\mathbf{F}_{\text {dep }}\left(R_{12}\right)$ as the difference between $\mathbf{F}_{2}\left(R_{12}\right)$ and the direct force $\mathbf{F}_{\text {dir }}\left(R_{12}\right)$ between the two stars due to their direct interaction potential $V_{11}\left(R_{12}\right)$. The magnitude of the depletion force $F_{\text {dep }}\left(R_{12}\right)$ is then given by

$$
F_{\text {dep }}\left(R_{12}\right)=\frac{\mathbf{R}_{12}}{R_{12}} \cdot \mathbf{F}_{\text {dep }}\left(R_{12}\right)
$$

Accordingly, the total effective interaction between the big star-polymers in a sea of the smaller species is the sum of their interaction potential $V_{11}(r)$ and the depletion potential $V_{\text {dep }}(r)$ :

$$
V_{\text {eff }}(r)=V_{11}(r)+V_{\text {dep }}(r)
$$

A large number of long simulation runs is required to to obtain accurate depletion forces with good statistics, which renders this approach inefficient if ones needs to calculate $V_{\text {eff }}(r)$ for arbitrary values of $q, f_{2}$ and $\rho_{2}^{\mathrm{r}}$. Thus, we resorted to approximative theoretical methods to calculate the effective interactions and used the simulation results at selected parameter combinations in order to put the theoretical approximations into test. The two theoretical approaches invoked in this work are the inversion of the Ornstein-Zernike equation and the superposition approximation, which are presented below. 


\section{B. Inversion of the Ornstein-Zernike equation}

The effective potential can be obtained by inversion of the two-component OZ equation results in the limit of low density of big stars [38, 52, 53]. It can be shown from diagrammatic expansions in the theory of liquids [57] that the radial distribution function $g(r)$ of any fluid whose constituent particles interact via the pair potential $V(r)$, reduces to the Boltzmann factor $g(r)=\exp [-\beta V(r)]$ in the low-density limit. The effective interaction between the big stars depends on the reservoir density $\rho_{2}^{\mathrm{r}}$ of the smaller component. The interaction can be obtained by solving the full two-component OZ equations with the RY closure for different small-component densities $\rho_{2}$ in the limit $\rho_{1} \rightarrow 0$; due to the latter limit, it then also holds $\rho_{2}=\rho_{2}^{\mathrm{r}}$. The radial distribution function $g_{11}(r)$ can then be inverted to yield the effective potential as

$$
\beta V_{\text {eff }}(r)=-\lim _{\rho_{1} \rightarrow 0} \ln \left[g_{11}\left(r ; \rho_{1}, \rho_{2}^{\mathrm{r}}\right)\right] .
$$

Thereafter, the depletion force $F_{\text {dep }}(r)$ can be calculated as $F_{\text {dep }}(r)=-\partial\left[V_{\text {eff }}(r)-V_{11}(r)\right] / \partial r$ and compared them to the simulation results of the preceding subsection. Selected comparisons are shown in Fig. 3, where it can be seen that the inversion of the OZ relation yields very reliable results. We emphasize here that the approximate character of the OZ-inversion technique lies exclusively in the approximations involved in solving the two-component integral equation theories, i.e., in the Rogers-Young (or any other chosen) closure relation. Otherwise, the method is based on the exact statement that the radial distribution function of a one-component system at low densities is equal to the Boltzmann factor of the associated pair potential and hence the agreement of the inversion method with the simulation results comes as no surprise. It rather corroborates the fact that the two-component RY-closure is very accurate whenever one deals with soft, repulsive interactions, a result already seen in the case of mixtures between hard spheres and star polymers [39].

\section{Superposition approximation}

Another possibility to derive the effective interaction is the superposition approximation (SA) [58]. If the density distribution $\rho_{2}\left(\mathbf{r} ; \mathbf{R}_{1}, \mathbf{R}_{2}\right)$ of the small stars around two big stars

held fixed at positions $\mathbf{R}_{1}$ and $\mathbf{R}_{2}$ is known, then the depletion force in the low-density limit can be calculated by a simple integration. The density $\rho_{2}\left(\mathbf{r} ; \mathbf{R}_{1}, \mathbf{R}_{2}\right)$ is proportional 
to three-body distribution function $g_{112}\left(\mathbf{R}_{1}, \mathbf{R}_{2}, \mathbf{r}\right)$, which expresses the probability density of finding a particle of species 2 at position $\mathbf{r}$, given that two particles of species one are fixed at positions $\mathbf{R}_{1}$ and $\mathbf{R}_{2}$. This function is in general unknown; a usual procedure is to approximate it by the product of pair distribution functions [58].

We consider two big stars at the positions $\mathbf{R}_{1}$ and $\mathbf{R}_{2}$ and choose, without loss of generality, $\mathbf{R}_{1}=0$. Let the distance between the particles be $R_{12}$. The surrounding smaller star-polymers have the density $\rho_{2}\left(\mathbf{r} ; \mathbf{R}_{1}, \mathbf{R}_{2}\right)$. By taking the average for fixed $R_{12}$ we obtain the depletion force as

$$
\begin{aligned}
F_{\text {dep }}\left(R_{12}\right)= & -2 \pi \int_{0}^{\infty} r^{2} \frac{\mathrm{d} V_{12}(r)}{\mathrm{d} r} \mathrm{~d} r \\
& \int_{-1}^{1} \rho_{2}\left(\mathbf{r} ; \mathbf{R}_{1}, \mathbf{R}_{2}\right) \omega \mathrm{d} \omega,
\end{aligned}
$$

where $\omega=\cos \theta$.

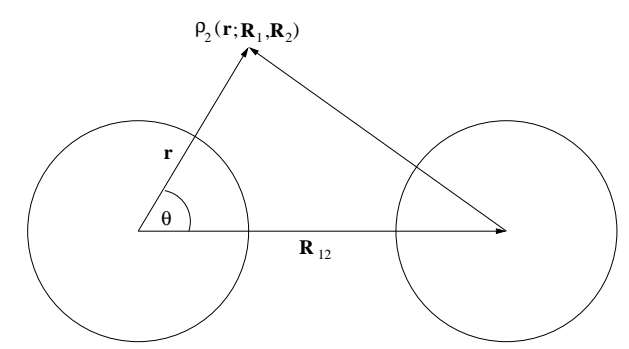

FIG. 4: A sketch of two big stars at a distance $R_{12}$ with $\rho_{2}\left(\mathbf{r} ; \mathbf{R}_{1}, \mathbf{R}_{2}\right)$ denoting the density of the smaller stars at $\mathbf{r}$. The density distribution depends on the positions of the two big star-polymers.

Since $\rho_{2}\left(\mathbf{r} ; \mathbf{R}_{1}, \mathbf{R}_{2}\right)$ is in general not known, at this point the exact density distribution has to be approximated. The density distribution around two big stars is replaced by the product of the density distributions around two isolated star-polymers at the positions $\mathbf{R}_{1}$ and $\mathbf{R}_{2}$, respectively. The SA then reads as

$$
\rho_{2}\left(\mathbf{r} ; \mathbf{R}_{1}, \mathbf{R}_{2}\right) \approx \rho_{2}^{\mathrm{r}} g_{12}\left(\left|\mathbf{r}-\mathbf{R}_{1}\right|\right) g_{12}\left(\left|\mathbf{R}_{2}-\mathbf{r}\right|\right),
$$

where $\rho_{2}^{\mathrm{r}}$ is the reservoir density, again identical to the system density for the situation at hand, since only two big stars are considered in the thermodynamic limit and thus $\rho_{1}=0$. The functions $g_{12}\left(\left|\mathbf{R}_{i}-\mathbf{r}\right|\right)$ are the radial distribution functions of small star polymers surrounding a single large one. Therefore, they can be obtained in the $\rho_{1} \rightarrow 0$-limit of the 
two-component OZ equations. Using simple geometrical considerations, we obtain $\left|\mathbf{r}-\mathbf{R}_{1}\right|=$ $\sqrt{R_{12}^{2}+r^{2}-R_{12} r \omega}$. Finally we obtain for the depletion force in the SA the expression:

$$
\begin{aligned}
F_{\text {dep }}\left(R_{12}\right) & =-2 \pi \rho_{2}^{\mathrm{r}} \int_{0}^{\infty} r^{2} \frac{\mathrm{d} V_{12}(r)}{\mathrm{d} r} g_{12}(r) \mathrm{d} r \\
& \times \int_{-1}^{1} g_{12}\left(\sqrt{R_{12}^{2}+r^{2}-R_{12} r \omega}\right) \omega \mathrm{d} \omega .
\end{aligned}
$$

The results we obtain by this method are compared to Monte Carlo and to those derived from inversion of the Ornstein-Zernike equation in Fig. 3. The results are very similar to the ones we obtain by inverting the $\mathrm{OZ}$ equation and both approximations yields reasonable agreement with the simulation data. Therefore, it is possible to choose the results of either approximation for calculating the phase diagrams and we expect that only minor quantitative differences will be seen by employing the one or the other theoretical approach. We have chosen to work with the effective interactions that result from the inversion of the OZequation since the latter is based on an exact statement, whereas the SA has an approximate nature.

\section{Effective interactions}

We have chosen to employ the effective interactions that result from the inversion of the OZ-equation since the latter is based on an exact statement, whereas the SA has an approximate nature. Representative results are shown in Fig. 5. For the lowest size ratio, $q=0.1$, we see that irrespective of the functionality of the depletants $\left(f_{2}=16\right.$ or 32$)$, the following scenario materializes: as $\rho_{2}^{\mathrm{r}}$ increases, first a weakening of the repulsions takes place, followed by the development of an attraction between the stars at sufficiently high reservoir densities, see Figs. 5(a) and 5(b). These findings provide a possible physical realization of the recently-proposed model ultrasoft repulsion potentials that are accompanied by an attractive part 59]. This attraction is more pronounced for $f_{2}=32$ than for $f_{2}=16$, if

one compares two systems with equal density $\rho_{2}^{\mathrm{r}} \sigma_{2}^{3}$. This result is not surprising, since the $f_{2}=32$-stars exert a higher osmotic pressure on the large ones than the $f_{2}=16$-stars and can therefore reduce the direct repulsions and induce attractions more efficiently.

Novel features in the effective potential appear for higher size ratios, $q=0.2$ and $q=0.3$. As can be seen in Fig. 馬(c)-(f), an oscillatory structure appears in the effective potential, 

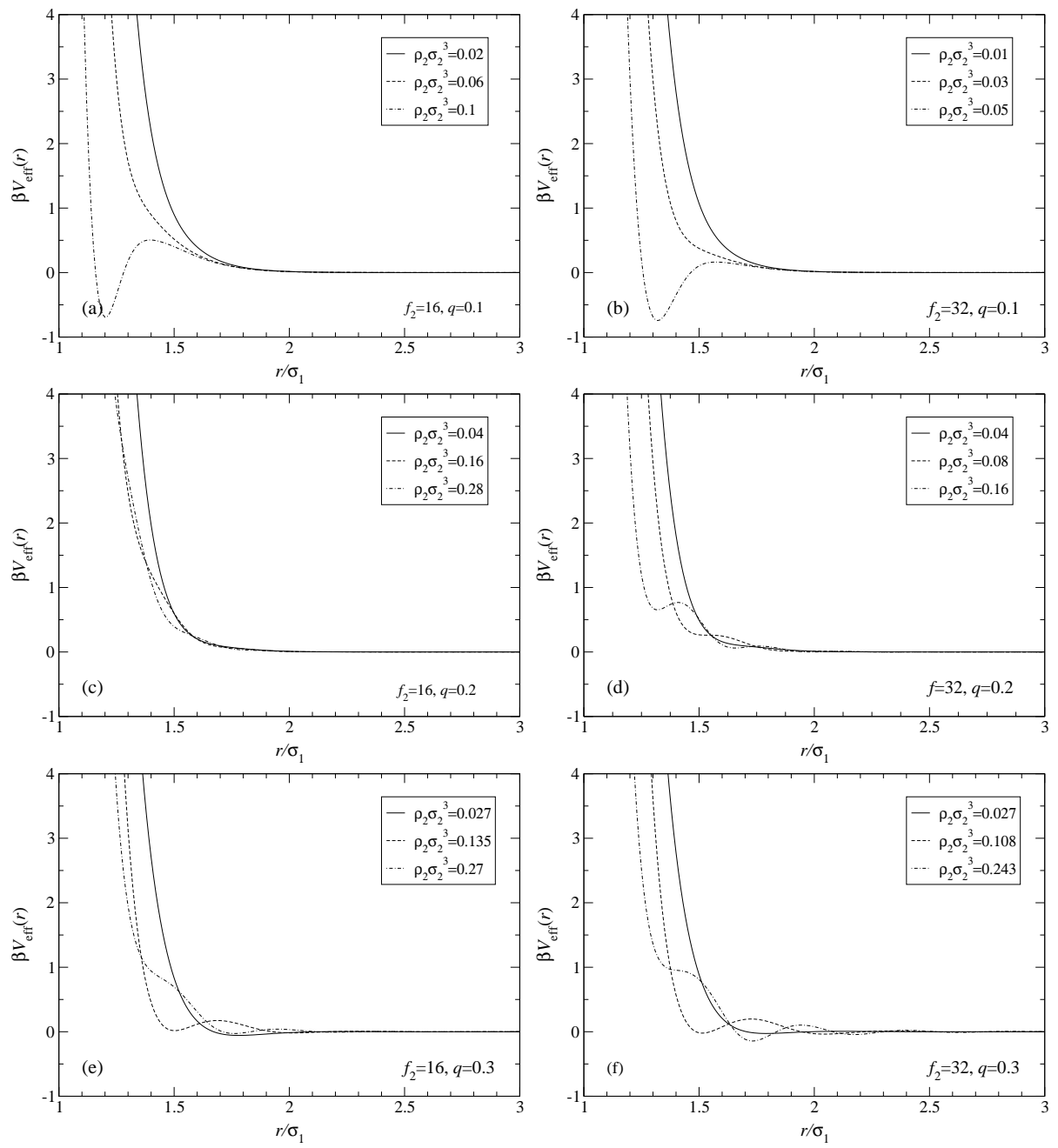

FIG. 5: The effective potential $V_{\text {eff }}(r)$ between two big star polymers in the presence of a sea of small ones. The various combinations of parameters regarding the density, functionality and size of the additives are shown in the legends. Notice the development of a strong attractive part in the interaction for the case $q=0.1$.

which is akin to that seen for hard-sphere mixtures of two different sizes. Contrary to this case, however, a deep attraction between the big stars does not develop and, therefore, it seems that a demixing transition between the two species does not exist when the sizes of the two stars become more and more similar. In all cases, however, the range of the repulsion decreases due to the depletion effect, i.e., the big star polymers appear, in the presence of the small ones, to be softer than they are in a pure solvent. Another possible interpretation, to be elaborated on in what follows, is that the big stars appear to be 'smaller', i.e., they 


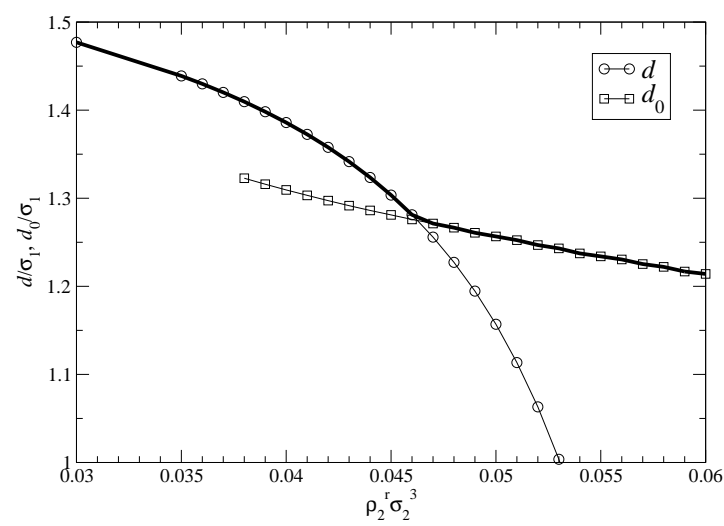

FIG. 6: The two possible effective hard sphere diameters $d$ and $d_{0}$ pertaining to the effective interaction $V_{\text {eff }}(r)$ between big stars, against the reservoir density $\rho_{2}^{\mathrm{r}}$. The parameter combination here is $f_{2}=32$ and $q=0.1$. As explained in the text, $d$ is calculated using the full effective interaction and $d_{0}$ only for the reference part.

acquire a reduced effective hard sphere packing fraction as a result of the depletants. Since the star-polymers then need less space, they become more mobile so the solid can melt. This property will be discussed in more detail in Sec. IVB.

\section{PHASE DIAGRAMS}

\section{A. Hard sphere mapping}

In order to trace out the phase diagram of the mixture in the $\left(\rho_{1}, \rho_{2}^{\mathrm{r}}\right)$-representation, we first perform a mapping of the effective one-component interaction $V_{\text {eff }}(r)$ between the big stars onto an effective hard-sphere system of diameter $\sigma$. Clearly, the latter depends on the reservoir density $\rho_{2}^{\mathrm{r}}$ as well as on the system parameters $q$ and $f_{2}$. For the purposes of performing the mapping in a physically meaningful way, we distinguish between two cases.

First, we consider the case in which $V_{\text {eff }}(r)$ is either free of attractive parts or positive definite or, at most, it contains negative parts not exceeding a small fraction of $k_{\mathrm{B}} T$ in magnitude. In this case, it is physically meaningful to identify $\sigma$ with the Barker-Henderson hard sphere diameter of the full effective interaction $V_{\text {eff }}(r), d$, defined as [60]:

$$
d=\int_{0}^{\infty}\left\{1-\exp \left[-\beta V_{\text {eff }}(r)\right]\right\} \mathrm{d} r .
$$

Most of the curves shown in Fig. 5 fall into this category. An important exception are the 
curves pertaining to $\rho_{2}^{\mathrm{r}} \sigma_{2}^{3}=0.1$ in Fig. 5 (a) and to $\rho_{2}^{\mathrm{r}} \sigma_{2}^{3}=0.05$ in Fig. [5 (b). For these combinations, and also for all others at even higher reservoir densities, a deep negative minimum appears in $V_{\text {eff }}(r)$ and application of Eq. (15) to such cases would lead to unphysically small and even negative effective hard sphere diameters. For such combinations, it is physically appealing to separate the effective potential $V_{\text {eff }}(r)$ into a purely repulsive part $V_{0}(r)$ and a perturbation part $V_{\text {pert }}(r)$, by truncating and shifting upwards the full interaction at the deepest minimum [60]. In this second case, it is pertinent to define another effective hard sphere diameter, $d_{0}$, that is associated with $V_{0}(r)$ only and is calculated again from the Barker-Henderson recipe, namely

$$
d_{0}=\int_{0}^{\infty}\left\{1-\exp \left[-\beta V_{0}(r)\right]\right\} \mathrm{d} r
$$

In attempting to choose and match between the two possible hard sphere diameters, $d$ and $d_{0}$, we are confronted with a technical difficulty. The evolution of the potential $V_{\text {eff }}(r)$ with $\rho_{2}^{\mathrm{r}}$ is continuous and the appearance of negative minima is in general accompanied by a soft repulsive barrier after the minimum. The effective hard sphere diameter, on the other hand, has to be a continuous function of $\rho_{2}^{\mathrm{r}}$, so as to avoid unphysical jumps of the phase boundaries in the phase diagram. In Fig. 6 we show a typical result for the dependence of $d$ and $d_{0}$ on $\rho_{2}^{\mathrm{r}}$. For low values of $\rho_{2}^{\mathrm{r}}$, where $V_{\text {eff }}(r)$ is purely repulsive, $d$ is a meaningful measure of the effective hard-sphere diameter. On the other hand, at high values of $\rho_{2}^{\mathrm{r}}$, where a deep attraction between the big stars effectively sets in, it is $d_{0}$ that most realistically captures the physics of the repulsions. The two curves cross at some point and, in order to guarantee both the continuity of $\sigma$ as a function of $\rho_{2}^{\mathrm{r}}$ and its correct asymptotic behavior for small and large values of $\rho_{2}^{\mathrm{r}}$, we choose

$$
\sigma=\max \left\{d, d_{0}\right\}
$$

It is then clear from the discussion above that for the perturbation part, $V_{\text {pert }}(r)$, of the interaction, it holds

$$
V_{\text {pert }}(r)=0 \quad \text { if } d>d_{0} .
$$

The phase diagrams can now be calculated using standard first-order perturbation theory 57] and taking Eqs. (17) and (18) into account. We do not take higher orders into account because we are mainly interested in the qualitative behaviour of the freezing line for small densities $\rho_{2}^{\mathrm{r}}$. Denoting by $F_{0}$ the Helmholtz free energy of the reference hard sphere system 
(effective hard sphere diameter $\sigma$ ), the total Helmholtz free energy $F$ of the one-component system consisting of $N_{1}$ big star polymers is approximated by

$$
\frac{\beta F}{N_{1}}=\frac{\beta F_{0}}{N_{1}}+\frac{1}{2} \beta \rho_{1} \int g_{0}(r) V_{\text {pert }}(r) \mathrm{d}^{3} r .
$$

In Eq. (19) above, $g_{0}(r)$ denotes the radial distribution function of the reference hard-sphere system in the fluid phase and its angle-averaged counterpart in the solid phase, as defined in Ref. [62].

We note here that more accurate methods for the treatment of potentials with a soft cores have also been proposed [61], but are not used here since we are only interested in the basic topology of the phase diagrams. For the free energy of the reference hard sphere system we used the equations of state of the Carnahan-Starling [63] and Hall [64] for the solid and fluid phases, respectively. For the calculation of pair distribution functions we use the expressions of Henderson and Grundke [65] for the fluid and of Kincaid and Weis [62] for the fcc-solid. We only considered the fcc-solid because this crystal structure appears at the fluid-solid transition in one component star-polymer solutions with an arm number $f_{1}=263[46]$.

\section{B. Results}

In Fig. 7 we show the resulting phase diagrams for size ratios $q=0.1$ and $q=0.2$ and functionalities of the smaller species $f_{2}=16$ and $f_{2}=32$, as obtained by the procedure described in the preceding subsection. The kinks for $q=0.1$ at about $\rho_{1} \sigma_{1}^{3}=0.8$ and $\rho_{2} \sigma_{2}^{3}=0.1$ and $\rho_{1} \sigma_{1}^{3}=0.6$ and $\rho_{2} \sigma_{2}^{3}=0.05$, respectively, are an artifact of the choice (17) and are associated with the sudden appearance of the $V_{\text {pert }}(r)$-term in Eq. (19), once we cross over from the case $d>d_{0}$ to the case $d<d_{0}$ (cf. also Fig. [6). Since we are primarily interested in the behavior for small densities $\rho_{2}^{\mathrm{r}}$ and the influence of the additives on crystallization, on the one hand, and on the possible existence of a spinodal line, on the other, a more sophisticated approach to the problem is at this stage not necessary. The liquid-solid coexistence region obtained by this approach is rather wide, due to the mapping on the effective hard-sphere system. Accurate calculations of the phase diagram of star polymers reveal that the coexistence region is much more narrow [46], yet the shape and evolution of the freezing lines as a function of $\rho_{2}^{\mathrm{r}}$ are not influenced by the width of the 

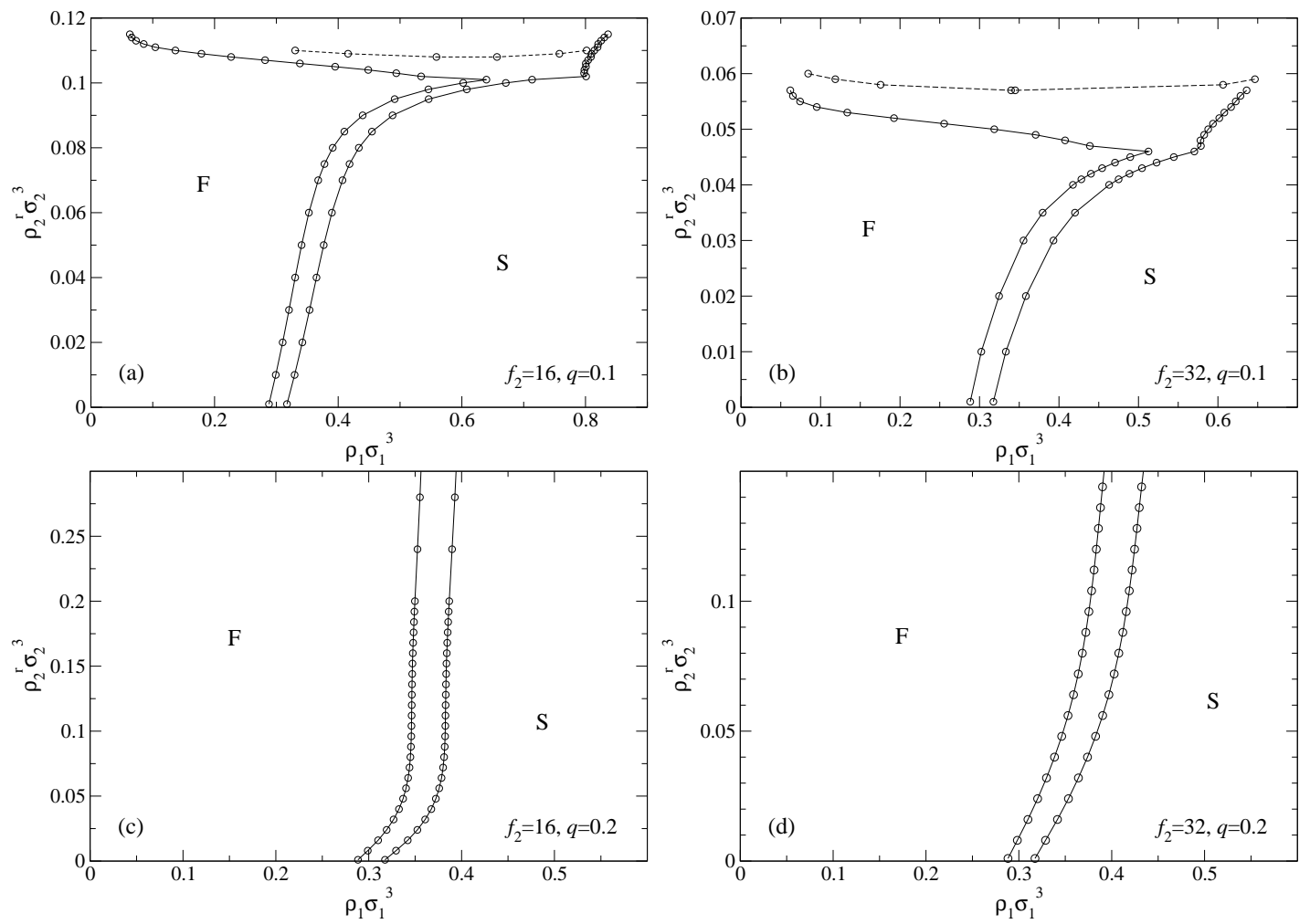

FIG. 7: Phase diagrams for star polymer mixtures for different parameter combinations. The big star functionality is fixed at $f_{1}=263$. The circles denote the phase boundaries as calculated by the hard-sphere mapping including the perturbation part $V_{\text {pert }}(r)$ and the lines are a guide to the eye. It can be seen that we only find a fluid-fluid demixing for $q=0.1$. This binodal line is metastable with respect to the formation of the fcc-solid. The kink in the phase boundaries for $q=0.1$ is an artifact and is caused by the method used to split the effective pair potential into reference and perturbation part. The symbol F stands for the fluid and the symbol S for the solid regions.

density gap between the fluid and the solid phases. Finally, we note that we have shifted the freezing line to higher densities by an amount $\Delta \rho_{1} \sigma_{1}^{3}=0.06$, in order to obtain the same density values for the crystallization as in the accurately known one component case [46].

For size ratio $q=0.1$, the effective potential $V_{\text {eff }}(r)$ develops a strong attraction. This leads to a broadening of the coexistence area between the solid and fluid phase and eventually to a demixing binodal. This binodal is found, however, to be metastable with respect to the crystallization. The sharp kinks that show up in Figs. Z(a) and Z(b) are artifacts of the way in which the effective hard sphere diameter $\sigma$ was determined and, in particular, of the fact that the attractive perturbation part $V_{\text {pert }}(r)$ of the effective potential is absent in the treatment 
for small reservoir densities below the kink and present above it. In reality, we expect the phase coexistence lines to 'turn around' smoothly, i.e., without the aforementioned artificial kink. However, the topology of the phase diagram and in particular the positive slope of the freezing lines and the subsequent broadening of the coexistence region into a 'gas-crystal' phase separation is not expected to be affected by these approximations. For larger values of $q$, a strong attraction does not emerge, so there is no fluid-fluid demixing. From Fig. (7) it can be seen that less stars with $f_{2}=32$ than with $f_{2}=16$ are needed to achieve similar effect. This is in agreement with the properties of star-polymer-colloid mixtures which were investigated in [39]. The metastable binodal is closer to the stable region of the phase diagram for the smaller functionality of the depletant. The same trends were also observed in star-polymer-colloid mixtures [39], where stable binodals were only found for small depletant functionalities such as $f_{2}=2$ and $f_{2}=6$. For the star-polymer mixtures we consider here, the existence of a binodal is less likely than in star-polymer-colloid mixtures, because for star polymers the depletion force has to overcome the Yukawa-type repulsion between them before an effective attraction sets in.

A striking effect is the melting of the crystal of the big stars upon addition of the small component, as can be seen from the positive slope of the freezing- and melting lines in Fig. 7. No effective attraction between the star-polymers is needed for this effect. As can be seen from Fig. 6, the effective hard sphere diameter $\sigma$ of the system decreases with increasing depletant density. Therefore, the effective packing fraction $\eta_{\mathrm{HS}}=(\pi / 6) \rho_{1} \sigma^{3}$ for the big stars gets smaller with increasing $\rho_{2}^{\mathrm{r}}$. Since this quantity determines the location of the freezing transition, the latter shifts to higher values of $\rho_{1}$. The melting is therefore caused by the apparent shrinkage of the stars due to the depletion effects. The counterintuitive behavior of melting of a crystal although the overall polymer concentration is increased has its physical roots in the soft depletion mechanism and the associated reduction of the range of the repulsive potential between the big stars.

In real experimental systems of star polymers, usually the formation of a glass is observed instead of crystallization. These glass transition lines are usually parallel to the freezing lines of the phase diagram, a property explicitly confirmed for the glass line of one-component star polymer solutions [50]. Therefore, we expect that the kinetic phase diagram of the mixture will have a topology running parallel to that of the equilibrium one that we traced in this study. We predict, therefore, that addition of small stars will bring about a melting 
of the colloidal glass (or gel) formed by the large ones. This has been shown to be the case for mixtures of star polymers with linear homopolymer chains [42].

\section{COMPARISON BETWEEN ONE- AND TWO-COMPONENT DESCRIP- TIONS}

\section{A. Chemical potentials}

By calculating the partial chemical potentials of the two-component system, we now map the reservoir representation $\left(\rho_{1}, \rho_{2}^{\mathrm{r}}\right)$ on the real physical system $\left(\rho_{1}, \rho_{2}\right)$, so as to make contact with experimental work, in which $\rho_{2}^{\mathrm{r}}$ has no direct relevance. The densities $\rho_{2}^{\mathrm{r}}$ and $\rho_{2}$ are linked by the condition that the chemical potential of the reservoir $\mu_{2}^{\mathrm{r}}$ has to be the same as the partial chemical potential in the system $\mu_{2}$.

The chemical potential in the reservoir of density $\rho_{2}^{\mathrm{r}}$ can easily be calculated if the fluid structure is known. The Helmholtz free energy density of the small stars in the reservoir, $F / V=f\left(\rho_{2}^{\mathrm{r}}\right)$, can be split into an ideal and an excess part:

$$
f\left(\rho_{2}^{\mathrm{r}}\right)=f_{\mathrm{id}}\left(\rho_{2}^{\mathrm{r}}\right)+f_{\mathrm{ex}}\left(\rho_{2}^{\mathrm{r}}\right)
$$

where $f_{\text {id }}(z)=\rho \ln \left(z \tau^{3}\right)-z$ and $\tau$ is an arbitrary length scale. The second derivative of the excess part is connected to the structure via the relation [66]

$$
f_{\mathrm{ex}}^{\prime \prime}\left(\rho_{2}^{\mathrm{r}}\right)=\frac{1}{\rho_{2}^{\mathrm{r}} S\left(k=0 ; \rho_{2}^{\mathrm{r}}\right)}-\frac{1}{\rho_{2}^{\mathrm{r}}},
$$

where $S(k)$ is the structure factor of the small stars in the reservoir (one-component system). The free energy can then be calculated by two integrations, with the integration constants determined by the conditions $f_{\mathrm{ex}}^{\prime}(0)=0$ and $f_{\mathrm{ex}}(0)=0$. The chemical potential of the reservoir is given by

$$
\mu_{2}^{\mathrm{r}}=f^{\prime}\left(\rho_{2}^{\mathrm{r}}\right)
$$

In order two calculate the partial chemical potential $\mu_{2}$ in the two-component system representation, we employ the so-called concentration structure factor [67]

$$
S_{\text {con }}(k)=x_{1} x_{2}^{2} S_{11}(k)+x_{1}^{2} x_{2} S_{22}(k)-\left(x_{1} x_{2}\right)^{3 / 2} S_{12}(k),
$$




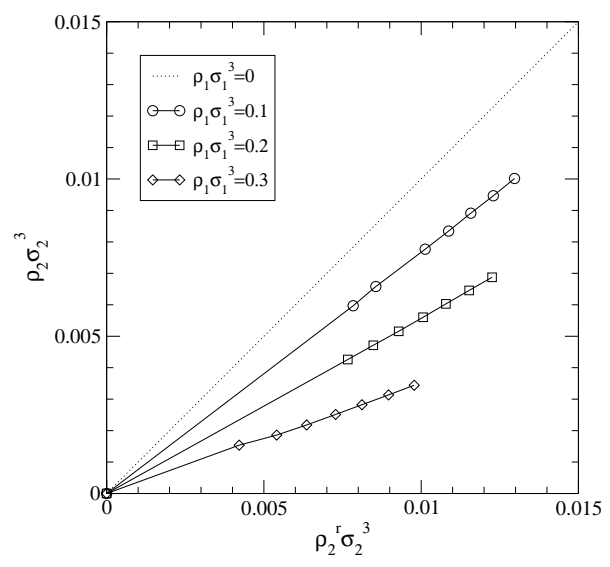

FIG. 8: Mapping between the system- and reservoir densities $\rho_{2}$ and $\rho_{2}^{\mathrm{r}}$ of the small stars for $f_{2}=32$ and $q=0.1$, and for various different values of the big star density $\rho_{1}$.

where $x_{i}=\rho_{i} /\left(\rho_{1}+\rho_{2}\right)$. Thermodynamic properties can then be calculated by using the equation [5, 67]

$$
\lim _{k \rightarrow 0} S_{\text {con }}(k)=\left[\frac{\partial^{2} g\left(x_{2}, P, T\right)}{\partial x_{2}^{2}}\right]^{-1},
$$

where $g\left(x_{2}, P, T\right)=G\left(x_{2}, N, P, T\right) / N$ is the Gibbs free energy per particle of the twocomponent mixture and $P$ its total pressure. Eq. (24) can then be integrated as described in Ref. [39] to yield the sought-for Gibbs free energy per particle $g\left(x_{2}, P, T\right)$. Once the Gibbs free energy is known, the partial chemical potentials can be calculated using the equations

$$
g^{\prime}\left(x_{2}\right)=\mu_{2}-\mu_{1}
$$

and

$$
g\left(x_{2}\right)-x_{2} g^{\prime}\left(x_{2}\right)=\mu_{1}
$$

Reverting from the pair of variables $\left(x_{2}, P\right)$ back to $\left(\rho_{1}, \rho_{2}\right)$ for the mixture and using Eqs 22, 25] and 26, the mapping $\left.\rho_{2}^{\mathrm{r}}\right) \rightarrow\left(\rho_{1}, \rho_{2}\right)$ can be carried out. Representative results are shown in Fig. 8. Clearly, the mapping depends parametrically on the big star density $\rho_{1}$ in the mixture. For $\rho_{1}=0$ one recovers $\rho_{2}=\rho_{2}^{\mathrm{r}}$. In all cases, we obtain $\rho_{2}<\rho_{s}^{\mathrm{r}}$ because all interactions are purely repulsive. The difference between the reservoir and system densities grows with increasing $\rho_{1}$. 

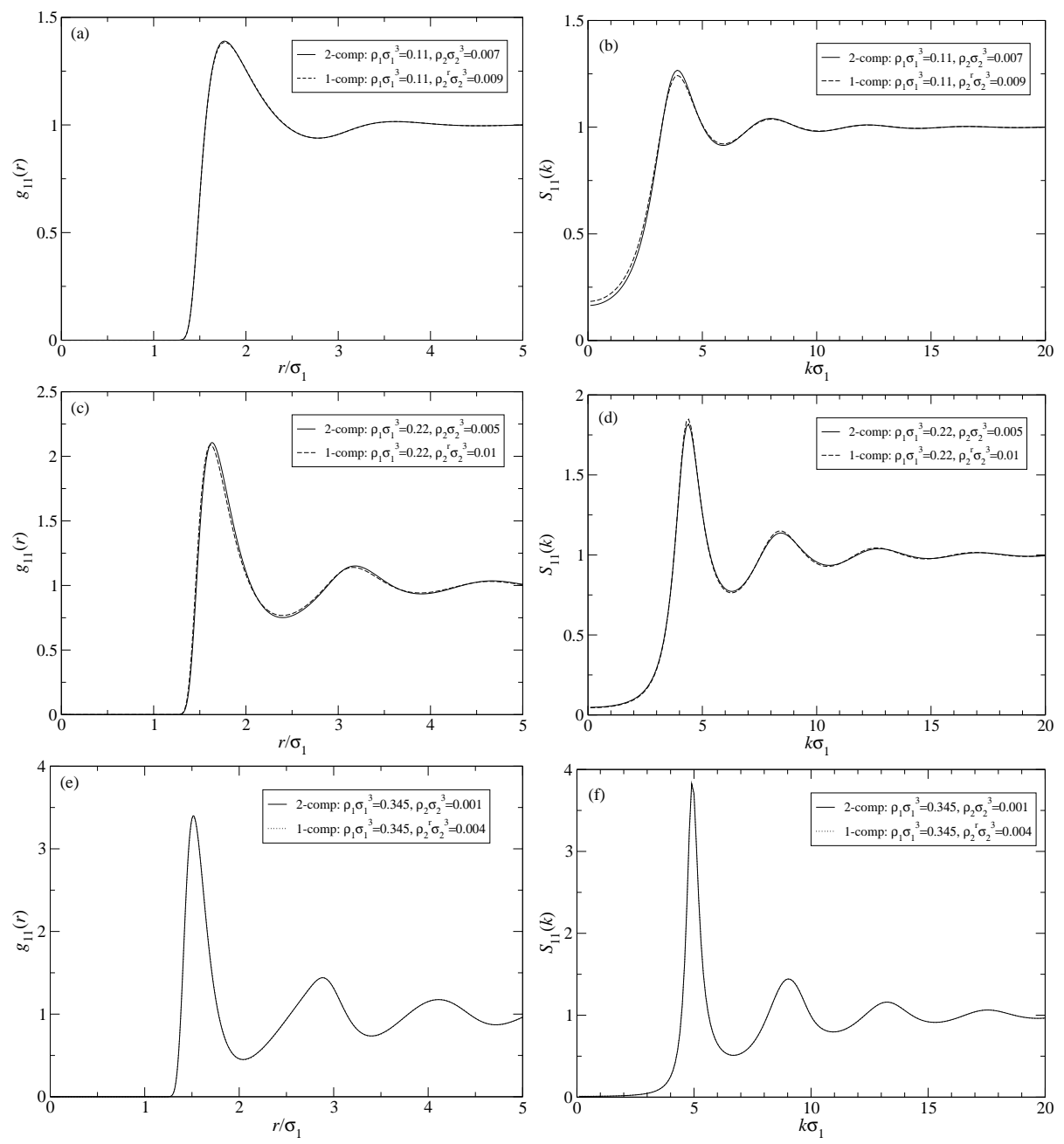

FIG. 9: Comparison of the fluid structure in the two- and effective one-component case. The densities are denoted in the diagrams. The structure of the mixture was calculated using the twocomponent RY closure, in the one component the usual RY closure was used. All plots are for $q=0.1$ and $f_{2}=32$. In panels (a), (e) and (f) the solid and dashed lines fall on top of each other, so that the latter cannot be distinguished from the former.

\section{B. Structure}

We now consider the spatial correlations between the big stars in the fluid phase. We compare the correlation functions calculated in the two-component description with those arising from the one-component description using the effective interactions in the presence of the smaller species. The comparison has to be carried out for parameter combinations such that the reservoir- and system partial chemical potentials of the smaller species are equal 
to one another. As the one-component description reduces the effective interaction to pair potentials, the comparison of the structural properties allows to estimate the magnitude of many body effects on the depletion.

In Fig. 9] we show the radial distribution functions and static structure factors of the big stars calculated with both different approaches. For the one-component case, the RY closure can be used because for the densities we consider here the effective interactions remain purely repulsive. The cases we consider here are for small densities $\rho_{2}$ but a wide range of values of $\rho_{1}$. The two component description includes many-body forces between the big stars which are caused by the smaller component. These effects are neglected in

the effective one-component description 9]. In Fig. 9, one can see excellent agreement of the results obtained by using these two different descriptions of the physical system. This additionally corroborates the validity of our approximation of the effective interaction. It can be also be concluded from the plots that the three-body forces are indeed much weaker than the pair interactions and can be safely neglected [68, 69].

\section{SUMMARY AND CONCLUSIONS}

We have analyzed the structural and phase behavior of highly asymmetric mixtures of star polymers, with the asymmetry characterizing both their sizes and functionalities. The most striking phenomenon predicted by our investigations is the counter-intuitive melting of the colloidal crystal of the big stars upon addition of small ones. Though this finding appears paradoxical at first sight, since addition of smaller stars increases the overall polymer concentration of the solution, its physical explanation can be traced to the effects of soft depletion. Whereas the depletants of hard spheres simply superimpose an effective attraction on a hard potential, when the big particles are themselves repulsive the depletion attraction is superimposed on a (soft) repulsion. In this way, a repulsive potential of reduced strength and/or range results and the effective, reduced repulsion is not any more sufficient to maintain the stability of the crystal, which therefore melts. When the depletant concentration becomes sufficiently high, the attractive depletion force dominates over the soft repulsion, leading to a (possibly metastable) demixing transition between the two species.

In real experimental systems of star polymers with high functionality, crystallization is hindered by the vitrification (gelation) transition and the large stars become structurally 
arrested in a glassy state above the overlap concentration. The next step would be then to investigate the role and influence of smaller star additives on the glass transition of larger stars, a problem of significant importance for the control of the rheological behavior of soft matter through additives. Work along these lines is currently under way and the presentation of the results of this investigation will be the subject of a future publication.

\section{Acknowledgments}

We thank Dimitris Vlassopoulos, Joachim Dzubiella, Martin Konieczny, Emanuela Zaccarelli, Francesco Sciortino, and Piero Tartaglia for helpful discussions. This work has been supported by the Marie Curie European Network MRTN-CT-2003-504712 and by the DFG within SFB TR 6.

[1] See, e.g., W. C. K. Poon, J. Phys.: Condens. Matter 14, R859 (2002) and references therein.

[2] S. Asakura and F. Oosawa, J. Chem. Phys. 22, 1255 (1954).

[3] S. Asakura and F. Oosawa, J. Polymer Sci. 33, 183 (1956).

[4] A. Vrij, Pure Appl. Chem. 48, 471 (1976).

[5] T. Biben and J.-P. Hansen, Phys. Rev. Lett. 66, 2215 (1991).

[6] T. Biben and J.-P. Hansen, J. Phys.: Condens. Matter 3, F65 (1991).

[7] M. Dijkstra, R. van Roij, and R. Evans, Phys. Rev. Lett. 81, 2268 (1998).

[8] M. Dijkstra, R. van Roij, and R. Evans, Phys. Rev. Lett. 82, 117 (1999).

[9] M. Dijkstra, R. van Roij, and R. Evans, Phys. Rev. E 59, 5744 (1999).

[10] See, e.g., M. Fuchs and K. S. Schweizer, J. Phys.: Condens. Matter 14, R239 (2002) and references therein.

[11] S. M. Ilett, A. Orrock, W. C. K. Poon, and P. N. Pusey, Phys. Rev. E 51, 1344 (1995).

[12] A. A. Louis, R. Finken, and J.-P. Hansen, Europhys. Lett. 46, 741 (1999).

[13] M. Dijkstra, J. M. Brader, and R. Evans, J. Phys.: Condens. Matter 11, 10079 (1999).

[14] M. Schmidt, H. Löwen, J. M. Brader, and R. Evans, Phys. Rev. Lett. 85, 1934 (2000).

[15] M. Fuchs and K. S. Schweizer, Europhys. Lett. 51, 621 (2000).

[16] M. Schmidt, H. Löwen, J. M. Brader, and R. Evans, J. Phys.: Condens. Matter 14, 9353 
(2002).

[17] M. Schmidt and M. Fuchs, J. Chem. Phys. 117, 6308 (2002).

[18] P. G. Bolhuis, A. A. Louis, and J.-P. Hansen, Phys. Rev. Lett. 89, 128302 (2002).

[19] For a recent review on inhomogeneous colloid-polymer mixtures, see J. M. Brader, R. Evans, and M. Schmidt, Mol. Phys. 101, 3349 (2003).

[20] J. M. Brader and R. Evans, Europhys. Lett. 49, 678 (2000).

[21] J. M. Brader, M. Dijkstra, and R. Evans, Phys. Rev. E 63, 041405 (2001).

[22] J. M. Brader, R. Evans, M. Schmidt, and H. Löwen, J. Phys.: Condens. Matter 14, L1 (2002).

[23] M. Dijkstra and R. van Roij, Phys. Rev. Lett. 89, 208303 (2002).

[24] W. K. Wijting, N. A. M. Besseling, and M. A. Cohen Stuart, Phys. Rev. Lett. 90, 196101 (2003).

[25] A. Moncho-Jorda, B. Rothenberg, and A. A. Louis, J. Chem. Phys. 119, 12667 (2003).

[26] P. P. F. Wessels, M. Schmidt, and H. Löwen, J. Phys.: Condens. Matter 16, L1 (2004).

[27] J. Bergenholtz and M. Fuchs, Phys. Rev. E 59, 5706 (1999).

[28] A. Johner, J.-F. Joanny, S. D. Orrite, and J. B. Avalos, Europhys. Lett. 56, 549 (2001).

[29] G. Foffi, K. A. Dawson, S. V. Buldyrev, F. Sciortino, E. Zaccarelli, and P. Tartaglia, Phys. Rev. E 65, 050802 (2002).

[30] A. M. Puertas, M. Fuchs, and M. E. Cates, Phys. Rev. E 67, 031406 (2003).

[31] J. Bergenholtz, W. C. K. Poon, and M. Fuchs, Langmuir 19, 4493 (2003).

[32] K. N. Pham, S. U. Egelhaaf, P. N. Pusey, and W. C. K. Poon, Phys. Rev. E 69, 011503 (2004).

[33] E. Zaccarelli, H. Löwen, P. P. F. Wessels, F. Sciortino, P. Tartaglia, and C. N. Likos, Phys. Rev. Lett., 92, 225703 (2004).

[34] C. N. Likos, Phys. Rep. 348, 261 (2001).

[35] A. A. Louis and R. Roth, J. Phys.: Condens. Matter 13, L777 (2001).

[36] B. Götzelmann, R. Roth, S. Dietrich, M. Dijkstra, and R. Evans, Europhys. Lett. 47, 398 (1999).

[37] R. Roth, R. Evans, and S. Dietrich, Phys. Rev. E 62, 5360 (2000).

[38] J. Dzubiella, C. N. Likos, and H. Löwen, Europhys. Lett. 58, 133 (2002).

[39] J. Dzubiella, C. N. Likos, and H. Löwen, J. Chem. Phys. 116, 9518 (2002).

[40] J. Dzubiella, A. Jusufi, C. N. Likos, C. von Ferber, H. Löwen, J. Stellbrink, J. Allgaier, D. Richter, A. B. Schofield, P. A. Smith, W. C. K. Poon, and P. N. Pusey, Phys. Rev. E 64, 
010401(R) (2001).

[41] A. A. Louis, E. Allahyarov, H. Löwen, and R. Roth, Phys. Rev. E 65, 061407 (2002).

[42] E. Stiakakis, D. Vlassopoulos, C. N. Likos, J. Roovers, and G. Meier, Phys. Rev. Lett. 89, $208302(2002)$.

[43] T. A. Witten and P. A. Pincus, Macromolecules 19, 2509 (1986).

[44] C. N. Likos, H. Löwen, M. Watzlawek, B. Abbas, O. Jucknischke, J. Allgaier, and D. Richter, Phys. Rev. Lett. 80, 4450 (1998).

[45] A. Jusufi, M. Watzlawek, and H. Löwen, Macromolecules 32, 4470 (1999).

[46] M. Watzlawek, C. N. Likos and H. Löwen, Phys. Rev. Lett. 82, 5289 (1999).

[47] C. von Ferber, A. Jusufi, M. Watzlawek, C. N. Likos, and H. Löwen, Phys. Rev. E 62, 6949 (2000).

[48] J. L. Lebowitz and J. S. Rowlinson, J. Chem. Phys. 41, 133 (1964).

[49] M. Watzlawek, H. Löwen, and C. N. Likos, J. Phys.: Condens. Matter 10, 8189 (1998).

[50] G. Foffi, F. Sciortino, P. Tartaglia, E. Zaccarelli, F. Lo Verso, L. Reatto, K. A. Dawson, and C. N. Likos, Phys. Rev. Lett. 90, 238301 (2003).

[51] J.-P. Hansen and L. Verlet, Phys. Rev. 184, 151 (1969).

[52] J. M. Méndez-Alcaraz and R. Klein, Phys. Rev. E 61, 4095 (2000).

[53] A. König and N. W. Ashcroft, Phys. Rev. E 63, 041203 (2001).

[54] I. D'Amico and H. Löwen, Physica A 235, 25 (1997).

[55] E. Allahyarov, I. D’Amico, and H. Löwen, Phys. Rev. Lett. 81, 1334 (1998).

[56] E. Allahyarov and H. Löwen, J. Phys.: Condens. Matter 13, L277 (2001).

[57] J.-P. Hansen and I. R. McDonald, Theory of Simple Liquids, 2nd ed. (Academic, London, 1986).

[58] P. Attard, J. Chem. Phys. 91, 3083 (1989).

[59] F. Lo Verso, M. Tau, and L. Reatto, J. Phys.: Condens. Matter 15, 1505 (2003).

[60] J. A. Barker and D. Henderson, J. Chem. Phys. 47, 4714 (1967).

[61] D. Ben-Amotz and G. Stell, J. Chem. Phys. 119, 10777 (2003).

[62] J. M. Kincaid and J. J. Weis, Mol. Phys. 34, 931 (1977).

[63] N. F. Carnahan and K. E. Starling, J. Chem. Phys. 51, 635 (1969).

[64] K. R. Hall, J. Chem. Phys. 57, 2252 (1970).

[65] D. Henderson and E. W. Grundke, J. Chem. Phys. 63, 601 (1975). 
[66] C. N. Likos and G. Senatore, J. Phys.: Condens. Matter 7, 6797 (1995).

[67] A. B. Bhatia and D. Thornton, Phys. Rev. B 2, 3004 (1970).

[68] S. Melchionna and J.-P. Hansen, Phys. Chem. Chem. Phys. 2, 3465 (2000).

[69] D. Goulding and S. Melchionna, Phys. Rev. E 64, 011403 (2001).

[70] The $\sigma_{i}$ are proportional to the corresponding radii of gyration, as described in Ref. 44]. 